Numerical Heat Transfer, Part A, 67: 1010-1027, 2015

Copyright (C) Taylor \& Francis Group, LLC

ISSN: $1040-7782$ print/1521-0634 online

DOI: $10.1080 / 10407782.2014 .955344$

\title{
EVALUATING THE OPTICAL PROPERTIES OF $\mathrm{TiO}_{2}$ NANOFLUID FOR A DIRECT ABSORPTION SOLAR COLLECTOR
}

\author{
Z. Said ${ }^{1,3}$, M. H. Sajid ${ }^{1}$, R. Saidur ${ }^{1,2}$, G. A. Mahdiraij ${ }^{4}$, and \\ N. A. Rahim ${ }^{2}$ \\ ${ }^{1}$ Department of Mechanical Engineering, Faculty of Engineering, University \\ of Malaya, Kuala Lumpur, Malaysia \\ ${ }^{2}$ Centre of Research UMPEDAC, Level 4, Engineering Tower, \\ Faculty of Engineering, University of Malaya, Kuala Lumpur, Malaysia \\ ${ }^{3}$ Department of Engineering Systems and Management (ESM), Masdar \\ Institute of Science and Technology, Abu Dhabi, United Arab Emirates \\ ${ }^{4}$ Department of Electrical Engineering, Faculty of Engineering, \\ University of Malaya, Kuala Lumpur, Malaysia
}

Recent studies specify that designated nanofluids may increase the proficiency of direct absorption solar thermal collectors. To determine the efficiency of nanofluids in solar applications, their capability to change light energy to thermal energy must be identified (i.e., the absorption spectrum of the solar material). In view of that, this study compares model predictions to spectroscopic measurements of extinction coefficients over waveleng ths that are important for solar energy $(200-1100 \mathrm{~nm})$. In the first decade of nanofluid research, most of the focus was on measuring and modeling the fundamental thermophysical properties of nanofluids (i.e., thermal conductivity, density, viscosity, and convection coefficients). Lately, considerable focus is given to the fundamental optical properties of nanofluids. However, the effect of particle size, shape, and volume fraction of nanoparticles as well as alternation of the base fluids, which can significantly affect scattering and absorption, have not been addressed to date in the literature. In this study, the effects of size and concentration of $\mathrm{TiO}_{2}$ nanoparticles on the extinction coefficient were analyzed using the Rayleigh approach. The results show that smaller particle size $(<20 \mathrm{~nm})$ has a nominal effect on the optical properties of nanofluids. Volume fraction is linearly proportionate to the extinction coefficient. Considering a nanoparticle size of $20 \mathrm{~mm}$, almost $0 \%$ transmissivity is obtained for wavelengths ranging from 200 to $300 \mathrm{~nm}$. However, a sudden increase of $71 \%$ in transmissivity is noted from $400 \mathrm{~nm}$, gradually increasing to $88 \%$ and becoming

similar to that of water at $900 \mathrm{~mm}$. Promising results are observed for volume fractions below $0.1 \%$.

Received 17 October 2013; accepted 13 August 2014.

Address correspondence to Z. Said, Department of Engineering Systems and Management (ESM), Masdar Institute of Science and Technology, P.O. Box 54224, Abu Dhabi, United Arab Emirates. E-mail: zaffar_14@yahoo.com; zsaid@masdar.ac.ae

Color versions of one or more of the figures in the article can be found online at www.tandfonline. com/unht. 


\begin{tabular}{|c|c|c|c|}
\hline \multicolumn{4}{|c|}{ NOMENCLATURE } \\
\hline$D$ & diameter of the particle & $M$ & relative complex refractive index of the \\
\hline$E$ & energy content & & nanofluid \\
\hline ETC & evacuated tube solar collector & $m_{p}$ & complex refractive index of the particle \\
\hline FPC & flat panel solar collector & $N$ & refractive index of the particle \\
\hline$f_{v}$ & volume fraction $(\%)$ & $Q$ & heat transfer \\
\hline$I$ & transmitted light intensity & $Q_{\text {abs }}$ & absorption coefficient \\
\hline$I_{\lambda}$ & total incident light intensity & $Q_{\text {ext }}$ & extinction coefficient \\
\hline$k$ & absorption index of the particle & $Q_{\text {scat }}$ & scattering coefficient \\
\hline$K_{\mathrm{a} \lambda}$ & spectral absorption coefficient & $R$ & distance between the particle and the \\
\hline$K_{\mathrm{e} \lambda}$ & spectral extinction coefficient & & observer \\
\hline$k_{f}$ & imaginary part of the complex refractive & $\Delta T$ & temperature difference \\
\hline & index & $\alpha$ & size parameter \\
\hline$K_{\mathrm{s \lambda}}$ & spectral scattering coefficient & $\theta$ & scattering angle \\
\hline$L$ & length of light path & $\lambda$ & wavelength $(\mu \mathrm{m})$ \\
\hline
\end{tabular}

\section{INTRODUCTION}

Researchers globally have devised and, in many cases, demonstrated interesting uses for dispersion of nanoparticles mixed or suspended in conventional fluids. Since the term nanofluid was coined by Choi [1] in 1995, researchers have found a wide variety of complex and innovative uses for this relatively simple class of nanotechnology. Numerous detailed reviews have been presented on nanofluid work in the thermal sciences field [1-15]. In order to achieve enhancement in nanofluids more careful measures need to be taken, as evidenced by modern research, to match the applications; in the case of optical properties of solar collectors this is particularly true. With very high volume fractions of nanoparticles it is observed that all incoming light is attenuated in a thin surface layer. Alternatively, with low volume fractions of nanoparticles, not all incoming solar radiation is attenuated. As a result, the optical properties of nanofluids must be precisely measured or inaccuracies in estimation may occur [16].

The concept of using a direct absorbing nanofluid, a liquid-nanoparticle suspension, has recently been shown numerically and experimentally to be an efficient method for harvesting solar thermal energy. A theoretical method and a novel computation algorithm were developed for the simulation of solar flat plate collectors with transparent insulation [17]. Tyagi et al. [18] investigated the feasibility of using a non-concentrating direct absorption solar collector (DASC) and compared its performance to a typical flat panel solar collector. The absorption of incident radiation was increased ninefold compared with pure water using $\mathrm{Al}_{2} \mathrm{O}_{3}$ nanofluid. For years the technology of nanofluids has been implemented in parabolic trough solar collectors. However, without the mirrors used to reflect and concentrate solar radiation in parabolic trough solar collectors, the fluid used for volumetric direct solar collectors ought to possess excellent optical properties beside the conventional requirement of good thermal transfer properties. Over the past decades, extensive research on the thermal behavior of nanofluids has been done and many correlations have been established and confirmed experimentally. However, slower progress in research of the optical properties of nanofluids has been reported. 
The solar-weighted absorption coefficient of fluid baseline capacity for absorbing solar energy was investigated and compared over four different liquids (i.e., water, ethylene glycol, propylene glycol, and therminol VP-1) [19]. Water absorbing only $13 \%$ of solar energy is found to be the best absorber for the solar-weighted absorption coefficient of fluid baseline [19]. On the other hand, improvement in collector efficiency has been made with the addition of small particles, which causes scattering of the incident radiation thereby enhancing absorption within the fluid [20]. Particle shape and size have a major impact on the optical properties of the effective fluid [21]. The concentration or volume/weight fraction of nanoparticles has a major impact on the optical absorption of nanofluid added to base fluid. Using nanofluids as a working medium for solar collectors is a relatively novel notion. With the availability of solar energy, researchers are interested in further developing the various aspects of this source of energy. Further investigations should be carried out for improving the physical properties of nanofluids to be used in direct solar collectors.

Titanium is used as a nanoparticle in this research as it is one of the most common and readily available metals. Besides, the thermal properties of titanium-based nanofluid have been studied extensively over the past decades and have shown favorable enhancements in optical properties. The effects of nanoparticles in enhancing optical properties are explored in this study, with nanoparticle size and volume fraction as variable parameters. The volume fraction of nanoparticles in the nanofluid should be lower to allow the potential to apply the results to evacuated tube solar collector (ETC) and to avoid shifting of the maximum absorption coefficient of nanofluid to a longer wavelength with less concentration.

This paper focuses on investigating the use of nanofluid as a volumetric absorber by studying the radiative properties of base fluid and nanoparticles, and the influence of nanoparticle size, nanofluid volume fraction, and transmissivity of light.

\section{RADIATIVE PROPERTIES OF NANOFLUID AND MODELING APPROACH}

Studies based on the optical properties of nanofluids show the need for separate investigation of the radiative properties of base fluid and nanoparticles. A brief theoretical explanation and the relation of light scattering and extinction for nanoparticles and base fluid are shown below. Long before nanofluid investigation became of predominant interest, scientists had focused on small particle absorption and scattering of light. Most of the recent theory of small particle optical properties was established over 100 years ago by many great scientists, including Gustav Mie. Remarkable overviews were presented on absorption and scattering [22-24]. A proficient theoretic foundation for the absorption and scattering of metallic small-scale particles was presented by Kreibig and Vollmer [25]. Predominantly in gas-particle mixtures, the radiative properties of small particles were introduced as being functional and were being used in other engineered systems by 1970 [19, 26-30]. Nanofluids have been studied for many years in regard to the perspective of their governing optical and radiative properties. The radiative and optical properties of nanofluids can be appreciably different from those of base fluid, and their dependent scattering effects will be of immense significance [31]. 
In the 1970s the first key concept of using particles for collecting solar energy was studied [26, 27], with particles being blended in a gas-operating fuel [32]. In subsequent years, particle recipients have been widely modeled and numerous prototype collectors have been built and tested. The volumetric absorption of radiation using particles has been experimentally demonstrated [26, 28, 33-36], where researchers blended particles with gas. Some recent studies however have shown the concept of liquid-based nanofluid solar collectors [3, 29, 37-45].

Small changes in regard to the size, shape, and volume fraction of nanoparticles, as well as alteration in base fluids, can result in significant improvement in scattering and absorption, which is of key importance in systems whose operating mode is nanoparticle suspension. A complete analysis of optical properties of the base fluid is required for understanding the effects of nanoparticles thereby suspended. The radiative properties of the fluid medium are important in regard to the efficiency of the volumetric design. An entirely transparent ideal fluid would be required for volumetric application for incoming solar radiation [46]. Nontoxic base fluids have to be used in direct solar collectors, such as the water heater. Water was found to be the best absorber among four tested liquids - water, ethylene glycol, propylene glycol, and therminol VP-1 [19] - by absorbing only $13 \%$ of the energy, but is still very weak absorber. The absorption and scattering properties of carbon nanohorn-based nanofluids for DASC using glycol and water as base fluid were studied by Mercatelli et al. [47], showing improvement over water-based nanofluids. Single-wall carbon nanohorns (SWCNH) were also investigated at the same concentration, and the enhancement of the thermal stability properties of the nanofluid by the optimization of the SWCNH-surfactant concentration ratio were observed. In the present study, water is selected as the base fluid while DASC is used mainly in residential development where the working temperature is estimated to be low. Scattering can be disregarded for pure fluids and only the attenuation caused by absorption may be considered. The spectral absorption coefficient can be calculated for the above case using Eq. (1):

$$
\sigma_{f}=\frac{4 \pi k_{f}}{\lambda}
$$

The absorption spectrum of pure water shows that water has a low absorption coefficient at shorter wavelengths that increases over the visible light spectrum until the near infrared region [48]. Figure 1 further illustrates the absorption coefficient of pure water, with refractive index and extinction/absorption index plotted versus wavelength [19]. The graphs compare the data from previous work [19, 49] based on numerical calculations. The refractive index of pure water contributes almost constant values from 200 to $1100 \mathrm{~nm}$, while the extinctive/absorptive index provides a marked increase and is reflected in the absorption coefficient as shown in Figure 1. Since the optical properties had long been studied and data have been verified by many researchers [19, 48-50], the spectrum of absorption coefficient used for calculation in this study is based on published data from previous works.

Suspensions of nanoparticles (i.e., particles of diameter $<100 \mathrm{~nm}$ ) in liquids, termed "nanofluids," show significant improvement in regard to thermal and optical property changes over the base liquid at low particle volume fractions [16]. 


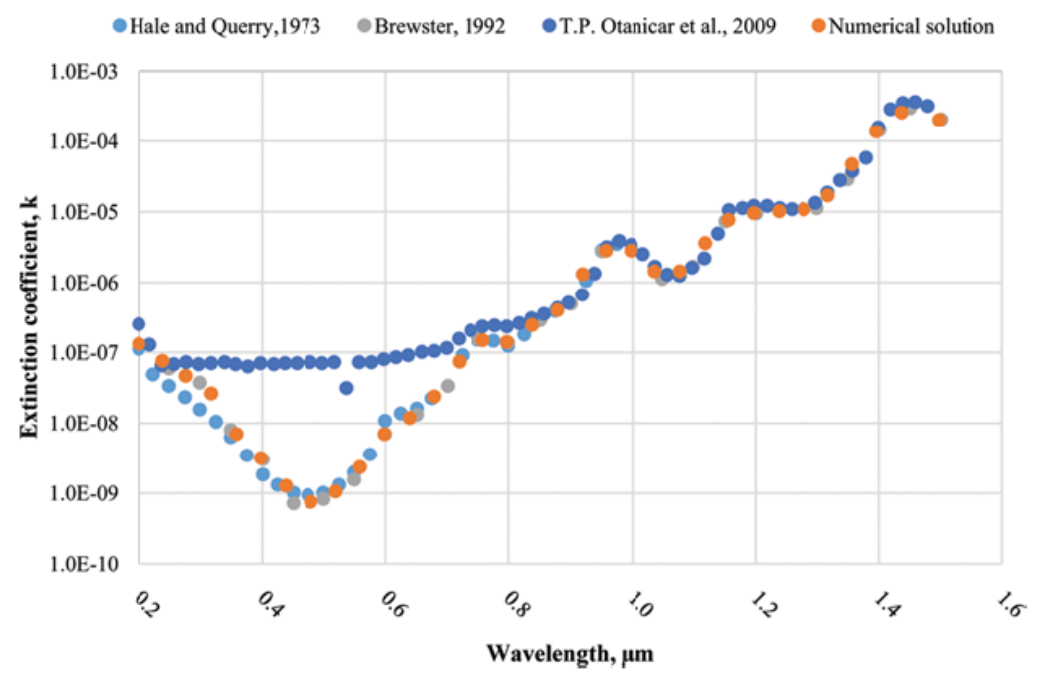

Figure 1. Experimental and calculated values of extinction index $(k)$ of water $[19,51,52]$.

Compared with thermal conductivity and convection studies on nanofluids, their optical and radiative properties have received much less attention. However, very recently the number of studies on radiative heat transfer in nanofluids has been growing [50]. This is due to the fact that, in general, the properties of a composite nanofluid are quite different from those of either the base fluid or the particles. At high temperatures, knowledge of the resultant radiative properties becomes increasingly important. Nanoparticles are only a small portion of the base fluid, with a small volume fraction and very small size. Therefore, the scattering effect of nanoparticles is considered independent in nanofluids. In reality, every particle is exposed to light scattered by the other particles, whereas the initial light beam might have endured by the other particles. Average particle diameter of commonly used nanoparticles is in the range $10-50 \mathrm{~nm}$, with most incident light from the sun having a wavelength that is at least 10 -fold greater. Therefore, scattering is mainly encountered using the Rayleigh scattering approach [38]. Due to the small size parameter $(\alpha<1)$, a simplified solution of the Rayleigh scattering is used, which is the Rayleigh approximation equations. The equations to calculate scattering $\left(Q_{\text {scat }}\right)$, absorption $\left(Q_{\text {abs }}\right)$, and extinction efficiencies $\left(Q_{\text {ext }}\right)$ of individual particles are as follows, and can be found in several standard texts such as Bohren and Huffman [18, 20, 24, 53-55]:

$$
\begin{gathered}
Q_{\text {scat }}=\frac{8}{3} \alpha^{4} \operatorname{Re}\left[\left(\frac{m^{2}-1}{m^{2}+2}\right)^{2}\right] \\
Q_{\text {abs }}=4 \alpha \operatorname{lm}\left\{\frac{m^{2}-1}{m^{2}+2}\left[1+\frac{\alpha^{2}}{15}\left(\frac{m^{2}-1}{m^{2}+2}\right) \frac{m^{4}+27 m^{2}+38}{2 m^{2}+3}\right]\right\} \\
Q_{\text {ext }}=Q_{\text {scat }}+Q_{\text {abs }}
\end{gathered}
$$


where $m$ is the relative complex refractive index of the nanofluid and $\alpha$ is the size parameter, which depends on particle diameter $D$ and incident wavelength $\lambda$ [56].

The relative complex refractive index of the nanofluid is given by [57]

$$
m=\frac{m_{p}}{n_{f}}
$$

Based on the extinction efficiency of nanoparticles, the extinction coefficient can be calculated using the following equation [55]:

$$
\sigma_{p}=\frac{3 \pi f_{v} Q_{\text {ext }}}{2 D}
$$

Scattering is usually insignificantly small. However, this is true only if the particles are uniformly small, while in practice some of the fluid may consist of larger particle agglomerates. Since scattering is ignored, the attenuated light is assumed to be absorbed and thus the extinction coefficient is simply the absorption coefficient [46]. The extinction coefficient of the base fluid can be calculated using Eq. (2). By combining the extinction coefficient of nanoparticles and base fluid we can calculate the total extinction coefficient of the nanofluids by the following equation:

$$
\sigma=\frac{3 \pi f_{v} Q_{\text {ext }}}{2 D}+\frac{4 \pi k_{f}}{\lambda}
$$

\subsection{Transmissivity of Light}

The radiative transport equation is used in this model. The right side of the equation determines the attenuation of intensity as radiation travels through the fluid. Equation (8) is integrated and expressed as [54]

$$
\begin{gathered}
\frac{\delta \boldsymbol{I}_{\lambda}}{\delta \boldsymbol{y}}=-\left(\boldsymbol{K}_{\mathrm{a} \lambda}+\boldsymbol{K}_{\mathrm{s} \lambda}\right) \boldsymbol{I}_{\lambda}=-\boldsymbol{K}_{\mathrm{e} \lambda} \boldsymbol{I}_{\lambda} \\
\boldsymbol{I}=\boldsymbol{I}_{\lambda} e^{-\boldsymbol{K}_{\mathrm{e} \lambda} y} \\
\frac{\boldsymbol{I}}{\boldsymbol{I}_{\lambda}}=e^{-\boldsymbol{K}_{\mathrm{e}, y} y}=T
\end{gathered}
$$

Equation (9) confirms that the intensity of light transmitted is inverse to the length of light path (y) and is further rearranged to create Eq. (10) to obtain transmissivity, $T$. This agrees with the Beer-Lambert law. Since temperatures in the solar collector are not expected to be very high, the emission term has not been included in Eq. (2). Also, in order to maintain the simplicity of the model, the effect of scattering has not been considered. 


\section{RESULTS AND DISCUSSION}

\subsection{Transmissivity of Base Fluid (Pure Water)}

The transmissivity $\boldsymbol{T}$ of the base fluid is first calculated to explore the effectiveness of light absorption for pure water. The transmissivity of pure water is shown in Figure 2. From this figure, pure water is seen to be almost transparent at the visible light region, and it absorbs none of the light transmitted to it. The absorption of light only becomes noticeable when light waves enter the near infrared region, and it reaches its maximum at $1.9 \mu \mathrm{m}$. Beyond $1.9 \mu \mathrm{m}$, pure water again becomes ineffective in absorbing light until the light wavelength exceeds $2.60 \mu \mathrm{m}$, when the absorption coefficient increases sharply and transmissivity falls abruptly. In general, pure water is not an effective light absorber especially in the visible light region. Thus, our work is focused on improving effective light absorption in the visible light region.

\subsection{Optical Properties of Nanoparticles}

Investigation of the effect of enhancing the optical properties of nanofluid with titanium nanoparticles starts with the selection of suitable nanoparticle size. As mentioned earlier, particle size is limited to under $100 \mathrm{~nm}$. The absorption coefficient scattering coefficient and extinction coefficient of titanium nanoparticles of size $1,5,10$, and $20 \mathrm{~nm}$ are calculated for volume fraction up to $0.08 \%$ using Eq. (1)-(7). The results are presented in Figures 3-13, which show the trend with increasing nanoparticle size.

Figures 3, 4, and 5 show the absorption coefficient, scattering coefficient, and extinction coefficient, respectively, of titanium nanoparticles versus light wavelength, with particle size as the variable parameter and a constant volume fraction of $0.05 \%$.

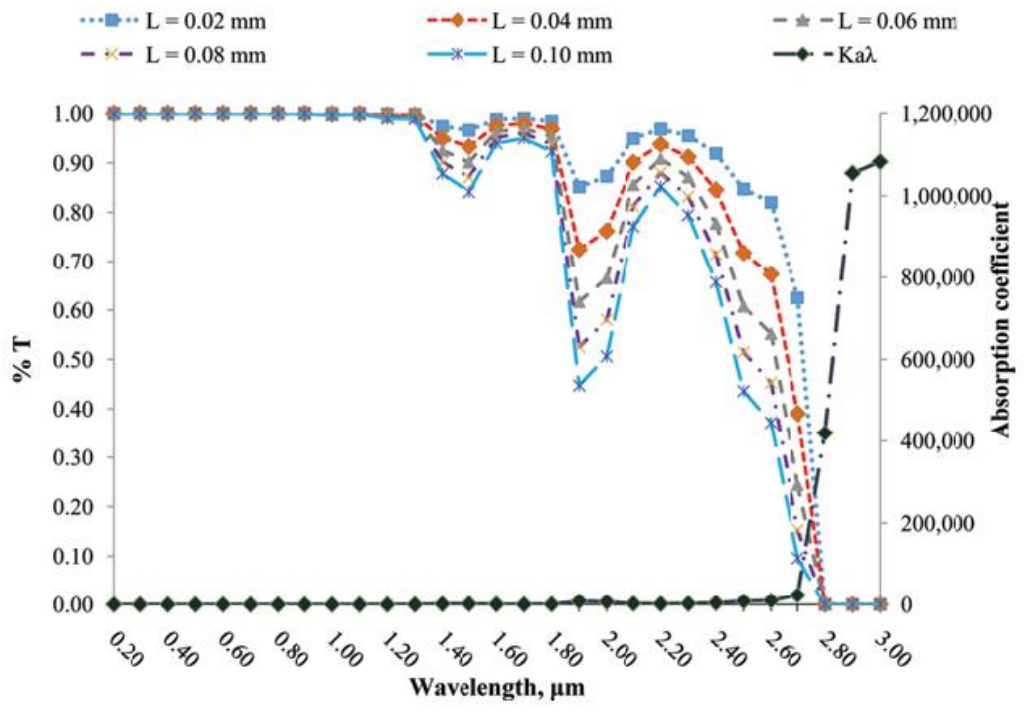

Figure 2. Transmissivity and absorption coefficient of pure water at various wavelengths [54] 


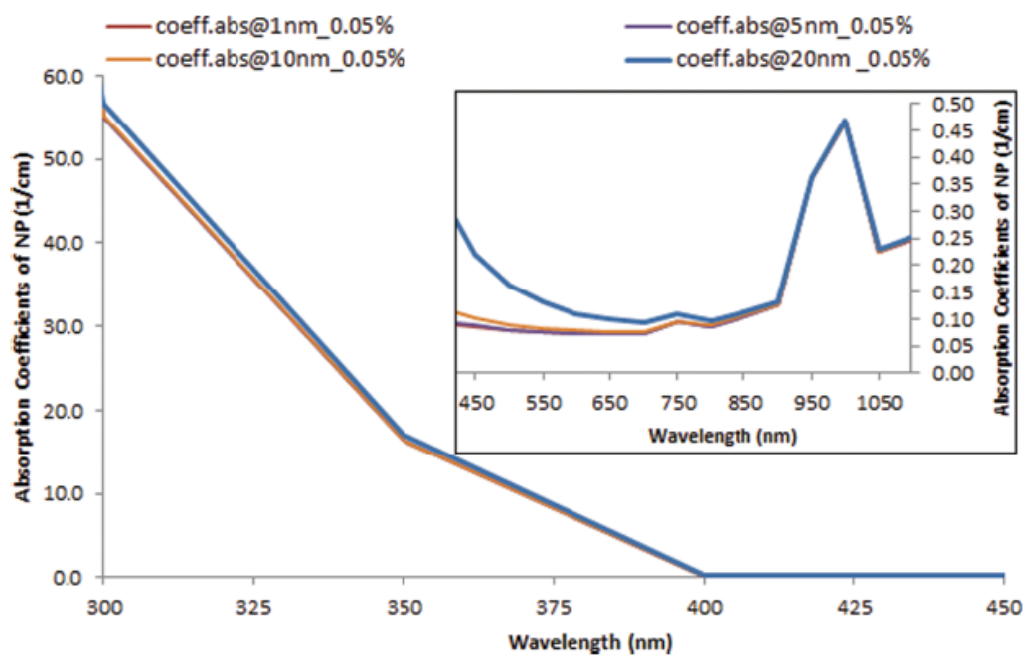

Figure 3. Effect of nanoparticle size on the absorption coefficient.

From Figure 3, it will be observed that particles under $5 \mathrm{~nm}$ will give almost the same absorption coefficient, as the curves for particle sizes of 1 and $5 \mathrm{~nm}$ overlap. With an increase in nanoparticle size, increase in absorption coefficient is noted. Higher absorption coefficient values are observed up to $400 \mathrm{~nm}$. Further increase in wavelength will overshadow the effect of particle size and eventually lead to very small values and a near-constant absorption coefficient, which is observed beyond $900 \mathrm{~nm}$. Figure 4 illustrates a different trend compared to that of the absorption

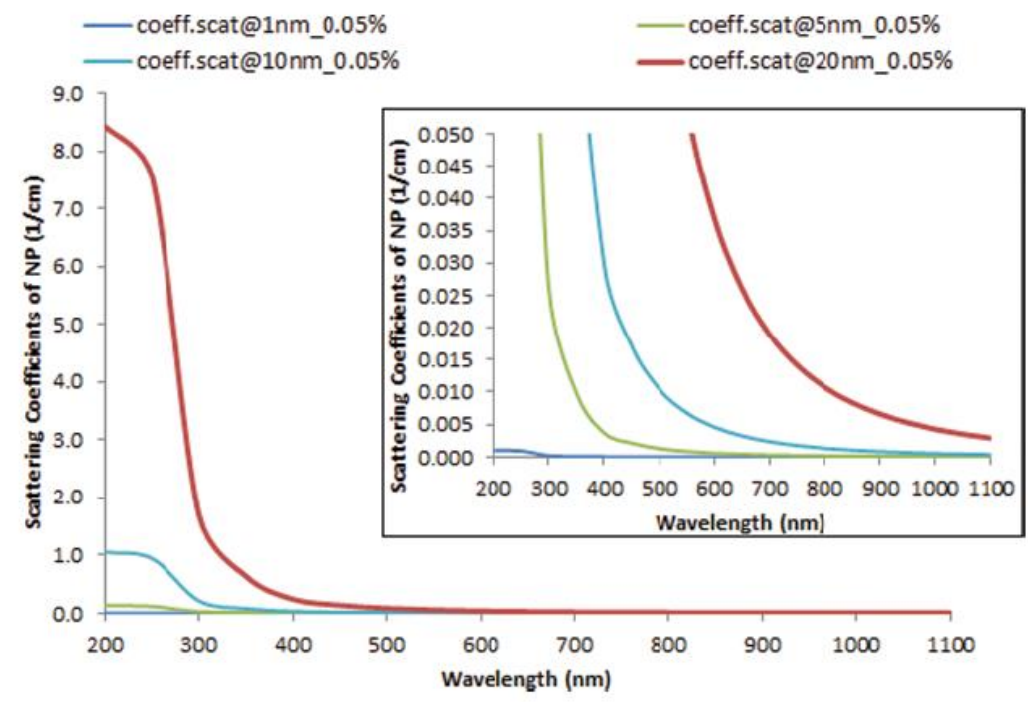

Figure 4. Effect of the nanoparticle size on the scattering coefficient. 


\section{Link to Full-Text Articles :}

http://www.tandfonline.com/doi/abs/10.1080/10407782.2014.955344\#.VRIar47fV5k 